Article

\title{
A Novel Population of Fusarium fujikuroi Isolated from Southeastern U.S. Winegrapes Reveals the Need to Re-Evaluate the Species' Fumonisin Production
}

\author{
Stephanie L. Bolton ${ }^{1}$, Phillip M. Brannen ${ }^{1}$ and Anthony E. Glenn ${ }^{2, *}$ \\ 1 Department of Plant Pathology, The University of Georgia, Athens, GA 30602, USA; \\ stephbolton@gmail.com (S.L.B.); pbrannen@uga.edu (P.M.B.) \\ 2 U.S. National Poultry Research Center, R.B. Russell Research Center, USDA-ARS, \\ Toxicology and Mycotoxin Research Unit, Athens, GA 30605, USA \\ * Correspondence: anthony.glenn@ars.usda.gov; Tel.: +1-706-546-3119 \\ Academic Editor: Gerhard Adam \\ Received: 11 July 2016; Accepted: 23 August 2016; Published: 31 August 2016
}

\begin{abstract}
Mycotoxins pose a challenge to a safe food supply worldwide, and their threat is expected to worsen with our changing climate. The need for diligence is exemplified by the discovery of fumonisin B2 in wine, which joins ochratoxin A as a mycotoxin of concern in the grape-wine chain. To elucidate the mycotoxin risk in southeastern American wine, grape samples were collected from vineyards during harvest in 2013 and potentially mycotoxigenic fungi (Fusarium and Aspergillus) were isolated from the samples. Numerous Fusarium isolates were recovered and identified to the species level by comparison of translation elongation factor $1-\alpha$ gene sequences to verified strains. Fusarium fujikuroi was the most abundant species recovered (239 isolates), followed by F. proliferatum (52), F. incarnatum-equiseti (14), F. oxysporum (7), F. concentricum (1), and F. solani (1). In vitro assays quantified fumonisin production for representative isolates via liquid chromatography-tandem mass spectrometry. Surprisingly, nearly all F. fujikuroi isolates produced fumonisins B1, B2, and B3 at levels comparable to both the F. proliferatum isolates and the positive control, Fusarium verticillioides. Such capacity for fumonisin production refutes the generally accepted notion that $F$. fujikuroi produces undetectable or low levels of fumonisins and provides evidence to reconsider this species as a mycotoxigenic threat to economically significant crops.
\end{abstract}

Keywords: fumonisin; Fusarium; Fusarium fujikuroi; grapes; ochratoxin A; Vitis vinifera; Fusarium proliferatum

\section{Introduction}

The possibility exists that mycotoxins, toxins produced by fungi having adverse effects on humans and animals, can survive the winemaking process to contaminate bottled wine even after the fungi themselves are eliminated from the must. The mycotoxin ochratoxin A (OTA) was discovered in 1965 by van der Merwe et al. [1], and the mycotoxins fumonisin B1 (FB1) and fumonisin B2 (FB2) were identified in 1988 [2]. In 1996, Zimmerli and Dick discovered OTA in wine [3] and more recently, in 2010 FB2 was revealed as a wine contaminant [4,5]. OTA, a mycotoxin produced by black-spored Aspergillus species such as Aspergillus carbonarius and Aspergillus niger [6-8], and present in cereals, wine, and coffee, has either been shown to be or is potentially nephrotoxic, immunosuppressive, teratogenic, genotoxic, embryotoxic, and cytotoxic [9]. OTA is also a possible human carcinogen in Group 2B, with fellow members dichlorodiphenyltrichloroethane (DDT), lead, and chloroform [10]. The source of FB2 in wine has been attributed to A. niger [11,12]. This is in contrast to FB1 in maize, which is the most common fumonisin in that commodity and is produced mainly by Fusarium verticillioides [13]. FB1 is 
noted as neurotoxic, hepatotoxic, and nephrotoxic in animals, and joins OTA as a possible Group 2B carcinogen [14].

Winegrapes are well established as a host for potentially mycotoxigenic black-spored aspergilli [15], but only a few reports have noted Fusarium species on winegrapes. One Slovakian survey revealed the presence of Fusarium proliferatum on grapes [16]. A second Slovakian survey isolated F. proliferatum from both surface-sterilized and non-sterilized grape berries, indicating that the fungus is capable of being present not only on the exterior of the grapes, but also within the berries [17]. A survey of Japanese wines found minor contamination (less than their limit of quantification, $1 \mathrm{ppb}$ ) with fumonisin B1 in a few samples [18] and later attributed this existence to F. proliferatum [19].

Only a few Fusarium species can produce the B series of fumonisins in significant concentrations, with $F$. verticillioides and F. proliferatum being the most notable. Despite having a fumonisin biosynthetic gene cluster (FUM) nearly identical to that of F. verticillioides and F. proliferatum [20], Fusarium fujikuroi is widely noted in the literature to produce low amounts, if any, of B-type fumonisins except in rare cases [21-25]. Interestingly, Suga et al. [26] found a single nucleotide polymorphism (SNP) in the translation elongation factor 1- $\alpha$ (TEF) gene of F. fujikuroi that correlated with minor fumonisin production in all Japanese isolates studied. This species is best known as a pathogen of rice contributing to Bakanae disease, or the "foolish seedling disease," caused by the gibberellin secondary metabolites produced by the fungus [27].

The southeastern American states currently producing commercially available red Vitis vinifera grapes are Alabama, Georgia, North Carolina, Tennessee, Kentucky, and Virginia. Popular grape varieties are Cabernet franc, Cabernet Sauvignon, and Merlot, although other varieties such as Sangiovese, Malbec, and Tannat are also grown. The Southeast represents a challenging environment for winegrapes, due to both disease and weather. Pierce's disease, vectored by the glassy-winged sharpshooter insect, is widespread, and a strict fungicide regimen is generally necessary to mitigate the effects of mildews and rots [28]. Weather challenges include late-season rains, hail, devastating spring frosts, and even hurricanes. Plant pathogens, insects, and poor weather may all contribute to a significant level of micro-punctured or wounded berries in the vineyard, which allow for easier access to berry nutrients by opportunistic fungal pathogens, such as black-spored Aspergillus and Fusarium spp. [29]. In fact, $A$. carbonarius produces OTA more efficiently on damaged berries than on intact ones [30]. Since winegrapes in the U.S. have the potential to be contaminated by mycotoxins, especially in the southeastern region where the climate is conducive to the growth of Aspergillus and Fusarium species, the presence of mycotoxigenic fungi in the vineyards was evaluated in the interest of public health safety and to fill a knowledge gap since vineyards within this region have not been extensively surveyed for these fungi. Thus, the objectives of this work were to isolate potentially mycotoxigenic fungi from southeastern U.S. red $V$. vinifera winegrapes, to identify these fungi to the species level, and to determine if they produce toxins in vitro. To the best of our knowledge, this study is the first report of $F$. fujikuroi isolated from winegrapes and the first report of a population of $F$. fujikuroi producing significant levels of fumonisins comparable to F. verticillioides and F. proliferatum.

\section{Results}

\subsection{Fungal Isolates Collected from Winegrapes}

A total of three black-spored Aspergillus isolates were recovered from the winegrape samples, each one from a different vineyard. None of these isolates had the morphological characteristics of the main OTA producer, A. carbonarius, and thus were not further investigated for mycotoxin production. In contrast, a total of 314 Fusarium isolates were recovered from the winegrape samples using dichloran rose bengal chloramphenicol (DRBC) and 2-benzoxazolinone (BOA) selective media. Based on sequencing of the translation elongation factor 1- $\alpha$ (TEF) region and BLASTN searches of three nucleotide databases for species identification, F. fujikuroi was the most abundant species recovered ( $n=239,76.1 \%$ of isolates), followed by F. proliferatum $(n=52,16.6 \%)$, F. incarnatum-equiseti $(n=14$, 
$4.5 \%)$, F. oxysporum $(n=7,2.2 \%)$, F. concentricum $(n=1,<1 \%)$, and F. solani $(n=1 ;<1 \%)$. F. fujikuroi isolates were recovered from every vineyard $(n=8)$ and every grape variety $(n=10)$ sampled (Table 1$)$, whereas F. proliferatum was recovered from fewer vineyards $(n=3)$ and grape varieties $(n=4)$, and only from the states of North Carolina and Alabama. The importance of collecting more than one isolate from a single sample replicate was demonstrated, as multiple species were found in distinct replicates.

Table 1. A high frequency of Fusarium fujikuroi isolates were recovered from southeastern winegrapes.

\begin{tabular}{cccccc}
\hline Vineyard & State & $\begin{array}{c}\text { \# of Sample } \\
\text { Replicates }\end{array}$ & $\begin{array}{c}\text { Total \# of } \\
\text { Fusarium } \text { Isolates }\end{array}$ & $\begin{array}{c}\text { \# of Fusarium } \\
\text { fujikuroi Isolates }\end{array}$ & $\begin{array}{c}\% \text { Fusarium } \\
\text { fujikuroi }\end{array}$ \\
\hline 01 & GA & 9 & 19 & 19 & $100 \%$ \\
02 & GA & 5 & 9 & 8 & $88.9 \%$ \\
05 & NC & 9 & 99 & 89 & $89.9 \%$ \\
09 & NC & 10 & 72 & 44 & $61.1 \%$ \\
12 & GA & 2 & 38 & 38 & $100 \%$ \\
$16^{\mathrm{b}}$ & AL & 3 & 43 & 7 & $16.3 \%$ \\
18 & GA & 9 & 28 & 28 & $100 \%$ \\
20 & GA & 9 & 6 & 6 & $100 \%$ \\
\hline
\end{tabular}

${ }^{a}$ One sample replicate is equivalent to two grape clusters. From each vineyard, three sample replicates were collected (where possible) from one to three different grape varieties; ${ }^{b}$ Vineyard 16 grape samples (the only non-vinifera variety, Norton) were collected approximately seven days post-harvest. All other samples were collected at commercial vineyards on the day of or a few days prior to harvest time.

To further support the determination that isolates were either F. fujikuroi or F. proliferatum, percent identity matrices were generated from ClustalW nucleotide alignments of winegrape isolates compared to multiple reference strains of each species. Identity of $\geq 97 \%$ was used to make species determinations, and F. fujikuroi was clearly distinguishable from F. proliferatum (Supplementary File). Also, due to the large number of isolates obtained from the winegrapes, only representative isolates from across vineyards, grape varieties, and sample replicates were deposited in the Agricultural Research Service (ARS) Culture Collection (NRRL), and the TEF sequences from these representatives were deposited in the National Center for Biotechnology Information (NCBI) GenBank (Table 2). Percent identity matrices of TEF alignments for those isolates not curated in NRRL and GenBank are presented in the Supplementary File as support of their species determination.

\subsection{In Vitro Fumonisin Production Analysis}

A representative group of F. proliferatum and F. fujikuroi winegrape isolates were further explored for their fumonisin producing capabilities in vitro using a rice-based assay (Table 2). Nearly all tested F. proliferatum isolates (20/22) produced fumonisins in the range of $1125-4653 \mathrm{ppm}(\mu \mathrm{g} / \mathrm{g})$ total fumonisins (FB1, FB2, and FB3). Average total fumonisins for F. proliferatum isolates was $1922 \mathrm{ppm}$. All F. fujikuroi isolates (50/50) produced fumonisins with an average total fumonisins of 1997 ppm (range of 1.09-3921 ppm). The average total fumonisins produced by the positive control, F. verticillioides, was $1675 \mathrm{ppm}$. No F. oxysporum isolates tested produced fumonisins in this study. There were no correlations with fumonisin production and state, vineyard, grape variety, or media isolation type (DRBC or BOA). Furthermore, isolates with the TEF SNP corresponding with non-production according to Suga et al. [26] did produce fumonisins in this study (Table 2). 
Table 2. In vitro fumonisin production by winegrape Fusarium isolates and F. verticillioides maize isolates.

\begin{tabular}{|c|c|c|c|c|c|c|c|c|c|c|c|}
\hline $\begin{array}{l}\text { Species Based } \\
\text { on TEF Seq. }\end{array}$ & $\begin{array}{l}\text { State (USA) } \\
\text { or Country }\end{array}$ & $\begin{array}{l}\text { Vineyard-Variety- } \\
\text { Replicate }^{\mathrm{a}}\end{array}$ & $\begin{array}{l}\text { Isolate No. } \\
\text { Assigned }\end{array}$ & $\begin{array}{l}\text { NRRL } \\
\text { No. }{ }^{b}\end{array}$ & $\begin{array}{c}\text { GenBank TEF } \\
\text { Accession }{ }^{b}\end{array}$ & $\begin{array}{l}\text { SNP } \\
\text { Type }^{c}\end{array}$ & $\begin{array}{c}\text { FB1 } \\
(\text { ppm) })^{d}\end{array}$ & $\underset{(\text { ppm })^{d}}{\text { FB2 }}$ & $\begin{array}{c}\text { FB3 } \\
(\text { ppm })^{d}\end{array}$ & $\begin{array}{c}\text { Total FB } \\
\text { (ppm) }\end{array}$ & $\begin{array}{c}\text { Fumonisin } \\
\text { Production }\end{array}$ \\
\hline F. concentricum & $\mathrm{NC}$ & 05-CF-1 & SLB 6283 & 66449 & KX711881 & - & ND & ND & ND & ND & ND \\
\hline F. fujikuroi & GA & 01-SA-2 & SLB 6163 & - & - & $\mathrm{Y}$ & 975 & 848 & 150 & 1973 & $\mathrm{HIGH}$ \\
\hline F. fujikuroi & GA & 01-SA-2 & SLB 6254 & 66445 & KX656201 & $\mathrm{Y}$ & 968 & 769 & 114 & 1850 & HIGH \\
\hline F. fuijkuroi & GA & 01-SA-2 & SLB 6184 & - & - & Y & 2403 & 1160 & 329 & 3891 & $\mathrm{HIGH}$ \\
\hline F. fuijkuroi & GA & 01-SA-3 & SLB 6185 & 66431 & KX656193 & Y & 1421 & 1194 & 301 & 2916 & HIGH \\
\hline F. fuijkuroi & GA & $01-\mathrm{TN}-2$ & SLB 6258 & - & - & $\mathrm{N}$ & 616 & 412 & 16 & 1043 & MEDIUM \\
\hline F. fujikuroi & GA & $01-\mathrm{TN}-3$ & SLB 6262 & 66446 & KX656202 & $\mathrm{Y}$ & 1706 & 327 & 139 & 2172 & HIGH \\
\hline F. fujikuroi & GA & $01-\mathrm{TN}-3$ & SLB 6270 & - & - & $\mathrm{Y}$ & 2336 & 524 & 265 & 3125 & HIGH \\
\hline F. fujikuroi & GA & 02-TA-2 & SLB 6309 & 66452 & KX656206 & $\mathrm{Y}$ & 1037 & 926 & 165 & 2128 & HIGH \\
\hline F. fujikuroi & GA & $02-\mathrm{TN}-2$ & SLB 6128 & 66427 & KX656189 & $\mathrm{Y}$ & 947 & 626 & 114 & 1687 & HIGH \\
\hline F. fujikuroi & $\mathrm{NC}$ & 05-CS-1 & SLB 6164 & 66428 & KX656190 & $\mathrm{Y}$ & 647 & 446 & 51 & 1144 & MEDIUM \\
\hline F. fujikuroi & NC & 05-CS-1 & SLB 6172 & - & - & $\mathrm{Y}$ & 720 & 566 & 84 & 1370 & MEDIUM \\
\hline F. fuijkuroi & $\mathrm{NC}$ & 05-CS-2 & SLB 6113 & 66425 & KX656187 & Y & 1482 & 1076 & 121 & 2678 & HIGH \\
\hline F. fujikuroi & NC & 05-CS-2 & SLB 6174 & - & - & $\mathrm{Y}$ & 1258 & 1116 & 261 & 2635 & HIGH \\
\hline F. fujikuroi & NC & $05-C S-3$ & SLB 6092 & - & - & $\mathrm{Y}$ & 1661 & 730 & 179 & 2570 & HIGH \\
\hline F. fujikuroi & NC & $05-C S-3$ & SLB 6118 & 66426 & KX656188 & $\mathrm{Y}$ & 1663 & 750 & 185 & 2598 & HIGH \\
\hline F. fujikuroi & NC & 05-CF-1 & SLB 6282 & 66448 & KX656204 & Y & 0.85 & 0.05 & 0.19 & 1.09 & LOW \\
\hline F. fujikuroi & NC & 05-CF-2 & SLB 6281 & 66447 & KX656203 & $\mathrm{Y}$ & 9 & 1640 & 1 & 1649 & HIGH-FB2 \\
\hline F. fujikuroi & NC & 05-ME-1 & SLB 6204 & - & - & $\mathrm{Y}$ & 1282 & 700 & 145 & 2126 & HIGH \\
\hline F. fujikuroi & NC & 05-ME-1 & SLB 6214 & 66439 & KX656197 & $\mathrm{Y}$ & 1275 & 711 & 149 & 2134 & HIGH \\
\hline F. fujikuroi & NC & 05-ME-2 & SLB 6224 & 66441 & KX656198 & $\mathrm{Y}$ & 1147 & 917 & 207 & 2271 & HIGH \\
\hline F. fujikuroi & NC & 05-ME-2 & SLB 6236 & 66444 & KX656200 & $\mathrm{Y}$ & 2 & 1728 & ND & 1730 & HIGH-FB2 \\
\hline F. fujikuroi & $\mathrm{NC}$ & 05-ME-3 & SLB 6234 & 66443 & KX656199 & $\mathrm{N}$ & 1961 & 455 & 49 & 2465 & HIGH \\
\hline F. fujikuroi & $\mathrm{NC}$ & 05-ME-3 & SLB 6239 & - & - & $\mathrm{N}$ & 1806 & 474 & 52 & 2331 & HIGH \\
\hline F. fujikuroi & $\mathrm{NC}$ & 05-ME-3 & SLB 6240 & - & - & $\mathrm{Y}$ & 1153 & 1022 & 282 & 2456 & HIGH \\
\hline F. fujikuroi & NC & 09-CS-1 & SLB 6020 & - & - & $\mathrm{Y}$ & 976 & 937 & 230 & 2142 & HIGH \\
\hline F. fujikuroi & NC & 09-CS-1 & SLB 6012 & 66415 & KX656183 & $\mathrm{Y}$ & 1627 & 1217 & 385 & 3229 & HIGH \\
\hline F. fujikuroi & $\mathrm{NC}$ & 09-CS-1 & SLB 6317 & - & - & $\mathrm{Y}$ & 1202 & 572 & 73 & 1846 & HIGH \\
\hline F. fujikuroi & NC & $09-\mathrm{CS}-3$ & SLB 6300 & - & - & $\mathrm{N}$ & 642 & 172 & 21 & 835 & MEDIUM \\
\hline F. fujikuroi & NC & $09-C S-3$ & SLB 6326 & 66453 & KX656207 & $\mathrm{N}$ & 826 & 209 & 13 & 1049 & MEDIUM \\
\hline F. fujikuroi & NC & 09-PN-1 & SLB 6023 & 66418 & KX656184 & $\mathrm{Y}$ & 1180 & 248 & 156 & 1584 & HIGH \\
\hline F. fuiikuroi & NC & 09-PN-1 & SLB 6005 & - & - & $\mathrm{Y}$ & 1055 & 420 & 164 & 1638 & HIGH \\
\hline F. fujikuroi & GA & $12-C S-1$ & SLB 6190 & - & - & $\mathrm{Y}$ & 1120 & 1028 & 283 & 2430 & HIGH \\
\hline F. fujikuroi & GA & 12-CS-1 & SLB 6196 & - & - & $\mathrm{Y}$ & 1046 & 958 & 248 & 2252 & HIGH \\
\hline F. fujikuroi & GA & 12-CS-1 & SLB 6177 & - & - & $\mathrm{Y}$ & 906 & 817 & 137 & 1861 & HIGH \\
\hline F. fujikuroi & GA & $12-C S-2$ & SLB 6140 & - & - & $\mathrm{Y}$ & 1815 & 830 & 175 & 2819 & HIGH \\
\hline
\end{tabular}


Table 2. Cont

\begin{tabular}{|c|c|c|c|c|c|c|c|c|c|c|c|}
\hline $\begin{array}{l}\text { Species Based } \\
\text { on TEF Seq. }\end{array}$ & $\begin{array}{l}\text { State (USA) } \\
\text { or Country }\end{array}$ & $\begin{array}{l}\text { Vineyard-Variety- } \\
\text { Replicate }^{\mathrm{a}}\end{array}$ & $\begin{array}{l}\text { Isolate No. } \\
\text { Assigned }\end{array}$ & $\begin{array}{l}\text { NRRL } \\
\text { No. }{ }^{b}\end{array}$ & $\begin{array}{c}\text { GenBank TEF } \\
\text { Accession } b\end{array}$ & $\begin{array}{l}\text { SNP } \\
\text { Type }\end{array}$ & $\underset{(p p m) d}{\text { FB1 }}$ & $\underset{(\mathrm{ppm})}{\mathrm{FB2}}$ & $\begin{array}{c}\text { FB3 } \\
(\text { ppm) }\end{array}$ & $\begin{array}{c}\text { Total FB } \\
\text { (ppm) }\end{array}$ & $\begin{array}{l}\text { Fumonisin } \\
\text { Production }\end{array}$ \\
\hline F. fujikuroi & GA & 12-CS-2 & SLB 6180 & 66429 & KX656191 & Y & 1520 & 1821 & 580 & 3921 & HIGH \\
\hline F. fujikuroi & $\mathrm{AL}$ & 16-NO-1 & SLB 6051 & 66421 & KX656185 & $\mathrm{Y}$ & 41 & 46 & 5 & 92 & LOW \\
\hline F. fujikuroi & $\mathrm{AL}$ & 16-NO-3 & SLB 6064 & - & - & $\mathrm{N}$ & 714 & 351 & 11 & 1076 & MEDIUM \\
\hline F. fujikuroi & $\mathrm{AL}$ & $16-\mathrm{NO}-3$ & SLB 6069 & 66423 & KX656186 & $\mathrm{Y}$ & 355 & 36 & 11 & 401 & LOW \\
\hline F. fujikuroi & GA & 18-MA-3 & SLB 6257 & - & - & $\mathrm{Y}$ & 1032 & 966 & 233 & 2231 & HIGH \\
\hline F. fujikuroi & GA & 18-CF-1 & SLB 6072 & - & - & $\mathrm{Y}$ & 1011 & 952 & 239 & 2201 & HIGH \\
\hline F. fujikuroi & GA & 18-CF-1 & SLB 6109 & - & - & $\mathrm{Y}$ & 1028 & 1029 & 221 & 2277 & HIGH \\
\hline F. fujikuroi & GA & 18-PV-1 & SLB 6278 & - & - & $\mathrm{N}$ & 840 & 390 & 29 & 1259 & MEDIUM \\
\hline F. fuijkuroi & GA & 18-PV-1 & SLB 6289 & 66450 & KX656205 & $\mathrm{N}$ & 574 & 268 & 12 & 853 & MEDIUM \\
\hline F. fuijkuroi & GA & 18-PV-1 & SLB 6284 & - & - & $\mathrm{N}$ & 720 & 347 & 25 & 1092 & MEDIUM \\
\hline F. fujikuroi & GA & 20-MA-2 & SLB 6203 & 66438 & KX656196 & $\mathrm{Y}$ & 1856 & 777 & 165 & 2798 & HIGH \\
\hline F. fujikuroi & GA & 20-MA-3 & SLB 6183 & 66430 & KX656192 & $\mathrm{Y}$ & 1348 & 899 & 179 & 2425 & HIGH \\
\hline F. fujikuroi & GA & 20-TA-1 & SLB 6199 & 66432 & KX656194 & $\mathrm{Y}$ & 1561 & 692 & 106 & 2359 & HIGH \\
\hline F. fujikuroi & GA & 20-TA-2 & SLB 6201 & 66437 & KX656195 & Y & 1502 & 550 & 143 & 2195 & HIGH \\
\hline F. fujikuroi & GA & 20-TA-2 & SLB 6200 & - & - & $\mathrm{Y}$ & 1402 & 509 & 123 & 2033 & HIGH \\
\hline F. oxysporum & GA & $02-\mathrm{TN}-1$ & SLB 6150 & - & - & $\mathrm{n} / \mathrm{a}$ & ND & ND & ND & ND & ND \\
\hline F. oxysporum & $\mathrm{NC}$ & 09-PN-1 & SLB 6007 & - & - & $\mathrm{n} / \mathrm{a}$ & ND & ND & ND & ND & ND \\
\hline F. oxysporum & NC & 09-ME-2 & SLB 6265 & - & - & $\mathrm{n} / \mathrm{a}$ & ND & ND & ND & ND & ND \\
\hline F. oxysporum & $\mathrm{AL}$ & $16-\mathrm{NO}-3$ & SLB 6063 & - & - & $\mathrm{n} / \mathrm{a}$ & ND & ND & ND & ND & ND \\
\hline F. proliferatum & NC & $05-C S-2$ & SLB 6099 & - & - & $\mathrm{n} / \mathrm{a}$ & ND & ND & 1723 & 1723 & HIGH-FB3 \\
\hline F. proliferatum & NC & 05-CS-2 & SLB 6100 & 66424 & KX656219 & $\mathrm{n} / \mathrm{a}$ & ND & $300^{f}$ & 3118 & 3418 & HIGH-FB3 \\
\hline F. proliferatum & $\mathrm{NC}$ & 05-CS-2 & SLB 6115 & - & - & $\mathrm{n} / \mathrm{a}$ & 3503 & 703 & 447 & 4653 & HIGH \\
\hline F. proliferatum & NC & 05-ME-2 & SLB 6228 & 66442 & KX656220 & $\mathrm{n} / \mathrm{a}$ & 1491 & 132 & 313 & 1936 & HIGH \\
\hline F. proliferatum & NC & 05-ME-2 & SLB 6237 & - & - & $\mathrm{n} / \mathrm{a}$ & 1681 & 150 & 326 & 2156 & HIGH \\
\hline F. proliferatum & NC & 09-CS-1 & SLB 6016 & 66417 & KX656215 & $\mathrm{n} / \mathrm{a}$ & ND & ND & ND & $\mathrm{ND}$ & ND \\
\hline F. proliferatum & $\mathrm{NC}$ & 09-CS-2 & SLB 6022 & - & - & $\mathrm{n} / \mathrm{a}$ & 1012 & 136 & 95 & 1243 & MEDIUM \\
\hline F. proliferatum & NC & 09-CS-2 & SLB 6013 & 66416 & KX656214 & $\mathrm{n} / \mathrm{a}$ & 1094 & 146 & 110 & 1349 & MEDIUM \\
\hline F. proliferatum & NC & $09-C S-2$ & SLB 6014 & - & - & $\mathrm{n} / \mathrm{a}$ & 985 & 136 & 101 & 1222 & MEDIUM \\
\hline F. proliferatum & NC & $09-C S-2$ & SLB 6322 & - & - & $\mathrm{n} / \mathrm{a}$ & 881 & 328 & 86 & 1295 & MEDIUM \\
\hline F. proliferatum & $\mathrm{NC}$ & 09-CS-2 & SLB 6298 & 66451 & KX656221 & $\mathrm{n} / \mathrm{a}$ & 771 & 294 & 61 & 1125 & MEDIUM \\
\hline F. proliferatum & $\mathrm{NC}$ & 09-CS-2 & SLB 6327 & - & - & $\mathrm{n} / \mathrm{a}$ & 971 & 368 & 98 & 1436 & MEDIUM \\
\hline F. proliferatum & NC & 09-CS-2 & SLB 6332 & - & - & $\mathrm{n} / \mathrm{a}$ & 937 & 352 & 91 & 1380 & MEDIUM \\
\hline F. proliferatum & $\mathrm{NC}$ & 09-PN-1 & SLB 6028 & - & - & $\mathrm{n} / \mathrm{a}$ & 1388 & 479 & 108 & 1974 & HIGH \\
\hline F. proliferatum & $\mathrm{NC}$ & 09-PN-1 & SLB 6009 & - & - & $\mathrm{n} / \mathrm{a}$ & 1488 & 497 & 110 & 2095 & HIGH \\
\hline F. proliferatum & $\mathrm{AL}$ & 16-NO-1 & SLB 6046 & - & - & $\mathrm{n} / \mathrm{a}$ & 1676 & 739 & 258 & 2673 & $\mathrm{HIGH}$ \\
\hline
\end{tabular}


Table 2. Cont

\begin{tabular}{|c|c|c|c|c|c|c|c|c|c|c|c|}
\hline $\begin{array}{l}\text { Species Based } \\
\text { on TEF Seq. }\end{array}$ & $\begin{array}{l}\text { State (USA) } \\
\text { or Country }\end{array}$ & $\begin{array}{c}\text { Vineyard-Variety- } \\
\text { Replicate }^{\text {a }}\end{array}$ & $\begin{array}{l}\text { Isolate No. } \\
\text { Assigned }\end{array}$ & $\begin{array}{l}\text { NRRL } \\
\text { No. }{ }^{b}\end{array}$ & $\begin{array}{c}\text { GenBank TEF } \\
\text { Accession }{ }^{b}\end{array}$ & $\begin{array}{l}\text { SNP } \\
\text { Type }^{c}\end{array}$ & $\begin{array}{c}\text { FB1 } \\
(\text { ppm) })^{d}\end{array}$ & $\begin{array}{c}\text { FB2 } \\
(\mathrm{ppm})^{\mathrm{d}}\end{array}$ & $\begin{array}{c}\text { FB3 } \\
(\text { ppm) })^{d}\end{array}$ & $\begin{array}{c}\text { Total FB } \\
\text { (ppm) }\end{array}$ & $\begin{array}{l}\text { Fumonisin } \\
\text { Production }\end{array}$ \\
\hline F. proliferatum & $\mathrm{AL}$ & 16-NO-1 & SLB 6042 & 66419 & KX656216 & $\mathrm{n} / \mathrm{a}$ & 2262 & 898 & 369 & 3528 & HIGH \\
\hline F. proliferatum & $\mathrm{AL}$ & 16-NO-1 & SLB 6045 & 66420 & KX656217 & $\mathrm{n} / \mathrm{a}$ & ND & ND & ND & ND & ND \\
\hline F. proliferatum & $\mathrm{AL}$ & 16-NO-2 & SLB 6038 & - & - & $\mathrm{n} / \mathrm{a}$ & 1886 & 244 & 138 & 2268 & HIGH \\
\hline F. proliferatum & $\mathrm{AL}$ & 16-NO-2 & SLB 6056 & 66422 & KX656218 & $\mathrm{n} / \mathrm{a}$ & 1642 & 242 & 90 & 1974 & HIGH \\
\hline F. proliferatum & $\mathrm{AL}$ & 16-NO-2 & SLB 6061 & - & - & $\mathrm{n} / \mathrm{a}$ & 2095 & 274 & 182 & 2550 & HIGH \\
\hline F. proliferatum & $\mathrm{AL}$ & 16-NO-2 & SLB 6052 & - & - & $\mathrm{n} / \mathrm{a}$ & 1880 & 270 & 127 & 2276 & HIGH \\
\hline F. verticillioides & CA & - & + control & 20956 & $\mathrm{n} / \mathrm{a}$ & $\mathrm{n} / \mathrm{a}$ & 663 & 104 & 26 & 793 & MEDIUM \\
\hline F. verticillioides & S. Africa & - & + control & 20960 & $\mathrm{n} / \mathrm{a}$ & $\mathrm{n} / \mathrm{a}$ & 956 & 499 & 10 & 1464 & MEDIUM \\
\hline F. verticillioides & IN & - & + control & 20984 & $\mathrm{n} / \mathrm{a}$ & $\mathrm{n} / \mathrm{a}$ & 1695 & 933 & 141 & 2769 & HIGH \\
\hline
\end{tabular}

a Identification system used in study. Two-digit codes were assigned to vineyards. Grape varieties are coded as follows: $\mathrm{CS}=\mathrm{Cabernet}$ Sauvignon, $\mathrm{CF}=\mathrm{Cabernet}$ Franc, ME $=\mathrm{Merlot}$ $\mathrm{MA}=$ Malbec, $\mathrm{NO}=$ Norton, $\mathrm{TA}=$ Tannat, $\mathrm{SA}=$ Sangiovese, $\mathrm{TN}=$ Touriga Nacional, $\mathrm{PN}=$ Pinot Noir, $\mathrm{PV}=$ Petit Verdot. The last digit refers to the replicate from which the isolate was obtained; ${ }^{b}$ A subset of representative isolates from this study were deposited in the Agricultural Research Service (ARS) Culture Collection (NRRL). The translation elongation factor (TEF) sequences of these curated isolates were deposited in the National Center for Biotechnology Information (NCBI) GenBank. Other winegrape isolates, indicated by a dash, were sequenced to confirm species identity, but sequences were not deposited in GenBank since the isolates were not curated in NRRL. $\mathrm{n} / \mathrm{a}=$ not applicable to $F$. verticillioides positive controls; ${ }^{\mathrm{c}} \mathrm{Y}=\mathrm{SNP}$ (single nucleotide polymorphism in TEF region) identified by Suga et al. [26] to be a fumonisin producer. N = SNP identified to be a non-producer. $\mathrm{n} / \mathrm{a}=$ the SNP applies to F. fujikuroi and is not applicable to other species; ${ }^{\mathrm{d}}$ Represents the average of a minimum of two independent experiments, each with three technical replicates Unit $=\mathrm{ppm}=\mu \mathrm{g}$ toxin per gram of dried rice. $\mathrm{ND}=$ none detected, limit of detection $(\mathrm{LOD})=0.013 \mathrm{ppb}$, and limit of quantification $(\mathrm{LOQ})=0.045 \mathrm{ppb}$; ${ }^{\mathrm{e}} \mathrm{ND}=\mathrm{none}$ detected LOW $=<500 \mathrm{ppm}$, MEDIUM $=500-1500 \mathrm{ppm}, \mathrm{HIGH}=>1500 \mathrm{ppm}$; ${ }^{\mathrm{f}} \mathrm{SLB} 6100$ is the only isolate tested which had discrepancy in production of FB2 between assays. In three of the four assay replicates (each tested in triplicate), this isolate produced no detectable FB2, but in one assay replicate it produced $1200 \mathrm{ppm}$ average FB2. 


\section{Discussion}

Whereas F. proliferatum is a well-known fumonisin producer, the general consensus in the Fusarium research community has been that $F$. fujikuroi produces little or no fumonisins. Surprisingly, all but two F. fujikuroi winegrape isolates tested with the rice assay produced significant levels of total fumonisins (greater than 400 ppm; either B1, B2, B3, or a combination). The levels of fumonisins produced by the F. fujikuroi winegrape isolates are comparable to the levels produced by the F. proliferatum winegrape isolates and to the F. verticillioides positive controls. This is the first report of a large population of F. fujikuroi isolates with the ability to produce high amounts of fumonisins.

Due to the rarity of a high fumonisin-producing F. fujikuroi population, further testing with verified F. fujikuroi and F. proliferatum isolates from the NRRL Culture Collection was conducted to determine if the rice assay method of in vitro fumonisin production, less common than the alternative cracked corn assay, was perhaps the cause of such production. One of these verified F. fujikuroi isolates, NRRL 31883, was isolated from a grape host in NY, USA. This one isolate, but none of the other NRRL F. fujikuroi reference strains we tested, produced high amounts of fumonisins similar to the southeastern winegrape isolates (Table 3). All other F. fujikuroi NRRL reference strains produced undetectable to low amounts of fumonisins $(<5 \mathrm{ppm})$. The species identity of all NRRL isolates tested was verified through sequencing of the TEF region as described earlier. Therefore, the rice assay testing method was not the cause of the high fumonisin production by F. fujikuroi winegrape isolates, which represent a truly unique population for this species. Differential regulation of the fumonisin biosynthetic gene cluster within this population may be the basis for the enhanced production of the mycotoxin. The grape host or the associated vineyard pest management practices may create an environment conducive to activation of the biosynthetic genes, perhaps related to nitrogen availability, which was shown to affect expression of the gene cluster [31]. Further studies would be needed to address these possibilities. We do wish to note that our laboratory has conducted fumonisin testing of southeastern American wines, and to date no wine samples have contained fumonisins at a level which would pose a consumer health risk (unpublished data).

Comparing published results of fumonisin production by Fusarium isolates is difficult due to the great diversity amongst in vitro fumonisin testing assays. Methods include a variety of growth media (cracked corn, rice, liquid broth, agar plates), incubation periods, toxin extraction methods, and means of reporting the toxin levels (ppm as $\mu \mathrm{g}$ toxin per gram of corn, $\mu \mathrm{g}$ toxin per gram dry fungal mass, etc.). Therefore, a more accurate and comprehensive approach is to obtain standard strains from a well-curated repository, verify their identity through sequencing, and test these strains in-house alongside collected experimental isolates. To this end, 35 of the winegrape Fusarium isolates were deposited into the permanent ARS Culture Collection. The NRRL numbers of these winegrape isolates are listed in Table 2. These strains may be useful to other researchers for in-house comparison of fumonisin production and genetic studies related to better understanding the F. fujikuroi secondary metabolite profile and its regulation. Given the wide host range of Fusarium species, this newly discovered high fumonisin-producing population of $F$. fujikuroi in vineyards in the Southeast raises concern for fumonisin contamination in other crops as well. Closer attention should be paid to the mycotoxigenic potential of $F$. fujikuroi worldwide. 
Table 3. In vitro fumonisin production by NRRL verified Fusarium strains.

\begin{tabular}{|c|c|c|c|c|c|c|c|c|c|}
\hline Species (TEF) & Source Host & Geographic Origin & NRRL ID ${ }^{a}$ & GenBank TEF Accession & FB1 (ppm) ${ }^{b}$ & FB2 (ppm) ${ }^{b}$ & FB3 (ppm) ${ }^{b}$ & Total FB (ppm) & Fumonisin Production $^{c}$ \\
\hline F. fujikuroi & sugarcane & Taiwan & 5538 & HF679028 & 3 & 0.6 & ND & 3.6 & LOW \\
\hline F. fujikuroi & rice & Taiwan & 13566 & AF160279 & ND & ND & ND & ND & ND \\
\hline F. fujikuroi & rice & Taiwan & 13998 & AB725605 & ND & ND & ND & ND & ND \\
\hline F. fujikuroi & rice & Taiwan & 22012 & JN092354 & 3.5 & ND & ND & 3.5 & LOW \\
\hline F. fujikuroi & soil & China & 31857 & KX656178 & ND & ND & ND & ND & ND \\
\hline F. fujikuroi & rice & GA, USA & 31862 & KX656179 & 3 & ND & ND & 3 & LOW \\
\hline F. fujikuroi & C tester strain & USA & 31879 & KX656180 & ND & ND & ND & ND & $\mathrm{ND}$ \\
\hline F. fujikuroi & grape & NY, USA & 31883 & KX656181 & 1334 & 1657 & 370 & 3360 & HIGH \\
\hline F. fujikuroi & rice & Nepal & 31886 & KX656182 & 2 & ND & ND & 2 & LOW \\
\hline F. proliferatum & flower & Germany & 22944 & AF160280 & 412 & 104 & 129 & 644 & MEDIUM \\
\hline F. proliferatum & soil & China & 31860 & KX656208 & 2770 & 650 & 190 & 3610 & HIGH \\
\hline F. proliferatum & fishtail palm & Australia & 31865 & KX656209 & 1225 & 136 & 74 & 1435 & MEDIUM \\
\hline F. proliferatum & peanut & GA, USA & 31866 & KX656210 & 1825 & 735 & 91 & 2650 & HIGH \\
\hline F. proliferatum & date palm & Iraq & 31867 & KX656211 & 1089 & 498 & 134 & 1721 & HIGH \\
\hline F. proliferatum & mango & Malaysia & 31871 & KX656212 & 945 & 621 & 87 & 1652 & HIGH \\
\hline F. proliferatum & Bakanae rice & Thailand & 31876 & none & 1448 & 165 & 148 & 1761 & HIGH \\
\hline F. proliferatum & rice & MS, USA & 31915 & KX656213 & 3589 & 653 & 319 & 4561 & HIGH \\
\hline F. verticillioides & rice & China & 22001 & AY337449 & 2680 & 752 & 132 & 3563 & HIGH \\
\hline F. verticillioides & maize & Germany & 22172 & AF160262 & 2008 & 925 & 118 & 3051 & HIGH \\
\hline
\end{tabular}

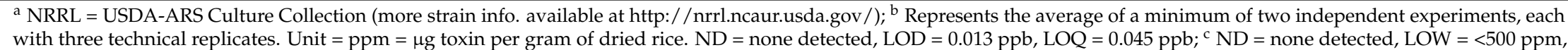
MEDIUM $=500-1500$ ppm, HIGH $=>1500$ ppm. 


\section{Materials and Methods}

\subsection{Collection of Winegrape Samples}

Samples of red, $V$. vinifera winegrapes were obtained from eight commercial vineyards in the southeastern U.S. (AL, GA, NC) during harvest in 2013. This harvest represented a vintage with both late season rains and high disease pressure. Grape varieties sampled included Cabernet Sauvignon, Cabernet franc, Malbec, Merlot, Petit Verdot, Pinot noir, Sangiovese, Tannat, and Touriga Nacional. One additional vineyard, planted with the non-vinifera variety of Norton, was also sampled. Up to three different grape varieties were sampled per vineyard. The majority of grape varieties sampled in each vineyard consisted of six clusters in total, each cluster randomly selected from a different non-adjacent grapevine on an individual vineyard row, forming up to three sample replicates of two clusters each. In total, 56 sample replicates were collected and processed. All clusters were brought to the laboratory on ice in re-sealable plastic bags and prepared for further analysis within $12 \mathrm{~h}$ from being cut from the vine. Stems were removed from clusters and grapes were homogenized (Polytron PT 10-35, Brinkmann Instruments, Thermo Fisher Scientific, Waltham, MA, USA) into a must slurry. The grapes were not surface sterilized in order to recover greater mycoflora diversity and to allow recovery of ochratoxigenic A. carbonarius or other black-spored Aspergillus species that typically sporulate on the grape berry exterior [15]. A sterile glycerin (Columbus Chemical Industries, Inc., Columbus, $\mathrm{OH}, \mathrm{USA}$ ) solution was added to grape must to a final concentration of $4 \%$, and the must solution was frozen at $-80{ }^{\circ} \mathrm{C}$ until further analysis.

\subsection{Fungal Isolation}

Thawed grape must sample replicates (1-3 per variety per vineyard) were serially diluted in sterile reverse osmosis (RO) water with $0.10 \%$ Tween ${ }^{\circledR} 20$ (Fisher Scientific, Fair Lawn, NJ, USA). Undiluted and serially diluted must samples were plated in duplicate onto two types of selective media: DRBC agar [32] and BOA agar [33]. Negative controls for each media type were included on every day of plating. Following a 5-7 day incubation period of DRBC plates at $30^{\circ} \mathrm{C}$ and a $7-10$ day incubation period of BOA plates at $27^{\circ} \mathrm{C}$, all potential black-spored Aspergillus and cottony-white Fusarium colonies were transferred onto potato dextrose agar (PDA) and incubated at $27^{\circ} \mathrm{C}$ or $30^{\circ} \mathrm{C}$ for purification and further identification. As Fusarium spp. were abundant, a maximum of 10 Fusarium colonies were isolated from each sample replicate and media type combination. Pure cultures of either Aspergillus or Fusarium were single-spore isolated via the dilution plating method on PDA [34]. Isolates were stored in $15 \%$ glycerin $/ 0.1 \%$ Tween ${ }^{\circledR} 20$ at $-80{ }^{\circ} \mathrm{C}$ for further analysis.

\subsection{DNA Extraction and Isolate Identification}

Single-spored Fusarium isolates were grown on PDA for $2-4$ days at $27{ }^{\circ} \mathrm{C}$ and genomic DNA was extracted using the previously described thermolysis procedure [35]. The primers EF-1 (5'-GTTAAGAGGCGCGGTGTCGGTGTG-3') and EF-2 (5'-GGAAGTACCAGTGATCATGTT-3') [36], along with $1 \mu \mathrm{L}$ of fungal genomic DNA and Taq Hot Start Quick-Load ${ }^{\circledR} 2 X$ Master Mix with Standard Buffer (New England BioLabs, Inc., Ipswich, MA, USA) were used to PCR-amplify a portion of the TEF gene for species identity [37]. PCR conditions were as follows: $94{ }^{\circ} \mathrm{C}$ for $30 \mathrm{~s}$, followed by 39 cycles of $94{ }^{\circ} \mathrm{C}$ for $10 \mathrm{~s}, 55^{\circ} \mathrm{C}$ for $10 \mathrm{~s}$, and $68^{\circ} \mathrm{C}$ for $45 \mathrm{~s}$, with a final extension of $68^{\circ} \mathrm{C}$ for $5 \mathrm{~min}$. Gene amplification was verified by gel electrophoresis, then PCR products were sent to GenScript USA, Inc. (Piscataway, NJ, USA) for purification and DNA sequencing. Sequencing primers used were the same as PCR primers. Sequences were edited with Sequencher ${ }^{\circledR}$ version 5.4.1 (Gene Codes Corporation, Ann Arbor, MI, USA). The TEF sequences were compared with verified strains in the Fusarium MLST (CBS-KNAW Fungal Biodiversity Centre, Utrecht, The Netherlands) [38], Fusarium-ID [39], and NCBI-BLAST [40] databases for species identification. Sequences from winegrape isolates were aligned to F. fujikuroi and F. proliferatum reference sequences from the databases using ClustalW implemented in Geneious analysis software (version 8.1.8, 2015, Biomatters Limited, Auckland, New Zealand) [41]. Percent nucleotide identity matrices were generated from the 
alignments, and an identity of $\geq 97 \%$ to reference isolates was used to designate winegrape isolates as either F. fujikuroi or F. proliferatum. Detailed phylogenetic characterization of these isolates will be published separately.

\subsection{In Vitro Fumonisin Production Analysis}

Following species identification, a subset $(n=73)$ of the total recovered potentially mycotoxigenic Fusarium winegrape isolates $(n=291)$ was selected for in vitro fumonisin analysis. This representative subset included both F. proliferatum $(n=22)$ and F. fujikuroi $(n=51)$ isolates from different states, vineyards, grape varieties, sample replicates, and media isolation types. Additionally, to test the Japanese isolate hypothesis that a SNP indicates fumonisin production or lack thereof, representative isolates with both producing and non-producing SNP types were included [26]. Four F. oxysporum winegrape isolates were assayed as well, though fumonisin B production is extremely rare for this species. Isolates of F. incarnatum-equiseti and F. concentricum, also from winegrapes, were included as negative controls. Additionally, strains of F. verticillioides, F. proliferatum, and F. fujikuroi were obtained from the USDA-ARS Culture Collection (NRRL) for inclusion in the fumonisin analysis (Peoria, IL, USA) [42]. Fumonisin-producing F. verticillioides strains (NRRL 20956, NRRL 20960, NRRL 20984) were used as positive controls, and one winegrape F. fujikuroi isolate (SLB 6326) that produced fumonisins in the first assay was used as an internal assay control for all subsequent assays. Therefore, each experimental run included testing potential fumonisin-producing Fusarium isolates from winegrapes and/or verified strains from the NRRL Culture Collection, a winegrape isolate negative control, a sterile water negative control, a F. verticillioides positive control, and an internal assay control. All isolates and strains analyzed, including controls, were tested in triplicate in each experiment. Assays were repeated on different days, and every winegrape isolate and verified strain was tested for fumonisin production in at least two independent experiments.

For fumonisin analysis, fungal isolates were grown on sterile rice using an assay modified from [27]. For each assay run, approximately $10 \mu \mathrm{L}$ of a high-inoculum spore suspension from frozen single-spored stock cultures $\left(-80{ }^{\circ} \mathrm{C}\right.$, in $15 \%$ glycerin $/ 0.1 \%$ Tween $\left.{ }^{\circledR} 20\right)$ was used as inoculum for $50 \mathrm{~mL}$ of sterile potato dextrose broth (PDB) in a $125 \mathrm{~mL}$ Erlenmeyer shaking flask. Culture flasks were incubated for 3 days $( \pm 1 \mathrm{~h})$ at $27{ }^{\circ} \mathrm{C}$ with shaking at $200 \mathrm{rpm}$ in darkness. To a $20 \mathrm{~mL}$ glass scintillation vial, $3 \mathrm{~g}( \pm 0.004 \mathrm{~g})$ of rice (enriched long grain rice, Kroger, Athens, GA, USA) and $3 \mathrm{~mL}$ of reverse osmosis water were added. Vials were topped with foam plugs, autoclaved $\left(121^{\circ} \mathrm{C}\right.$, $30 \mathrm{~min}$ ), and cooled to room temperature just prior to use. In triplicate, $250 \mu \mathrm{L}$ of high inoculum spore suspension ( $\sim 10^{7}$ spores per $\left.\mathrm{mL}\right)$ from incubated PDB flasks (or sterile water control) were added to each rice vial replicate. Inoculated rice vials were incubated for 7 days $( \pm 1 \mathrm{~h})$ at $27^{\circ} \mathrm{C}$ in darkness.

Following the incubation period on rice, $10 \mathrm{~mL}$ of a 1:1 acetonitrile (HPLC grade, Fisher, Fair Lawn, NJ, USA): distilled water extraction solution containing 5\% formic acid ( 98\%, Sigma-Aldrich, St. Louis, MO, USA) was added to each vial. Samples were vigorously shaken and frozen to $-20^{\circ} \mathrm{C}$ for at least $10 \mathrm{~h}$. Prior to LC-MS/MS analysis for fumonisins, rice cultures were broken apart and vials were rocked for $3 \mathrm{~h}$ (The Belly Dancer ${ }^{\circledR}$, speed 6.5, Stovall Life Science, Inc., Greensboro, NC, USA) to extract any fumonisins. The resulting extraction solution was centrifuge-filtered $\left(\operatorname{costar}^{\circledR}\right.$ Spin-X HPLC Micro Centrifuge Filter, $0.45 \mu \mathrm{m}$, nylon, Corning Inc., Corning, NY, USA), diluted (up to 10,000 fold), and analyzed via LC-MS/MS (Thermo LTQ XL). All analyses were multiple reaction monitoring (MRM) performed in positive ion mode, and the instrument was tuned using FB1 (PROMEC, Tygerberg, South Africa). An Imtakt Cadenza CW-C18 column $(150 \times 2$ mm, $3 \mu \mathrm{m}$; Kyoto, Japan), maintained at $30{ }^{\circ} \mathrm{C}$, was used for separations. Solvent A was distilled water with $3 \%$ acetonitrile plus $0.1 \%$ formic acid, and solvent $\mathrm{B}$ was acetonitrile with $3 \%$ distilled water plus $0.1 \%$ formic acid. The solvent program began at 30:70 A:B, and increased linearly to 100\% B over 9 min, held at $100 \%$ B for $2 \mathrm{~min}$, and then returned to starting conditions. Samples were run along with a blank control and standard solutions of fumonisins B1, B2, and B3 (1 ppb, 10 ppb, and 100 ppb in 30:70 acetonitrile:distilled water plus $0.1 \%$ formic acid; PROMEC, Tygerberg, South Africa). Analytes were identified by retention time, as compared to standards, and fragmentation patterns (Figure 1). Levels 
of fumonisins were quantified by external calibration of peak areas (the MS/MS total ion current) with standards, and a new calibration curve (limit of quantification (LOQ) $=0.045 \mathrm{ppb}$ and limit of detection $(\mathrm{LOD})=0.013 \mathrm{ppb}$ ) was run with each sample set. Fumonisin amounts were reported as $\mu \mathrm{g}$ toxin per gram of dried rice (ppm).
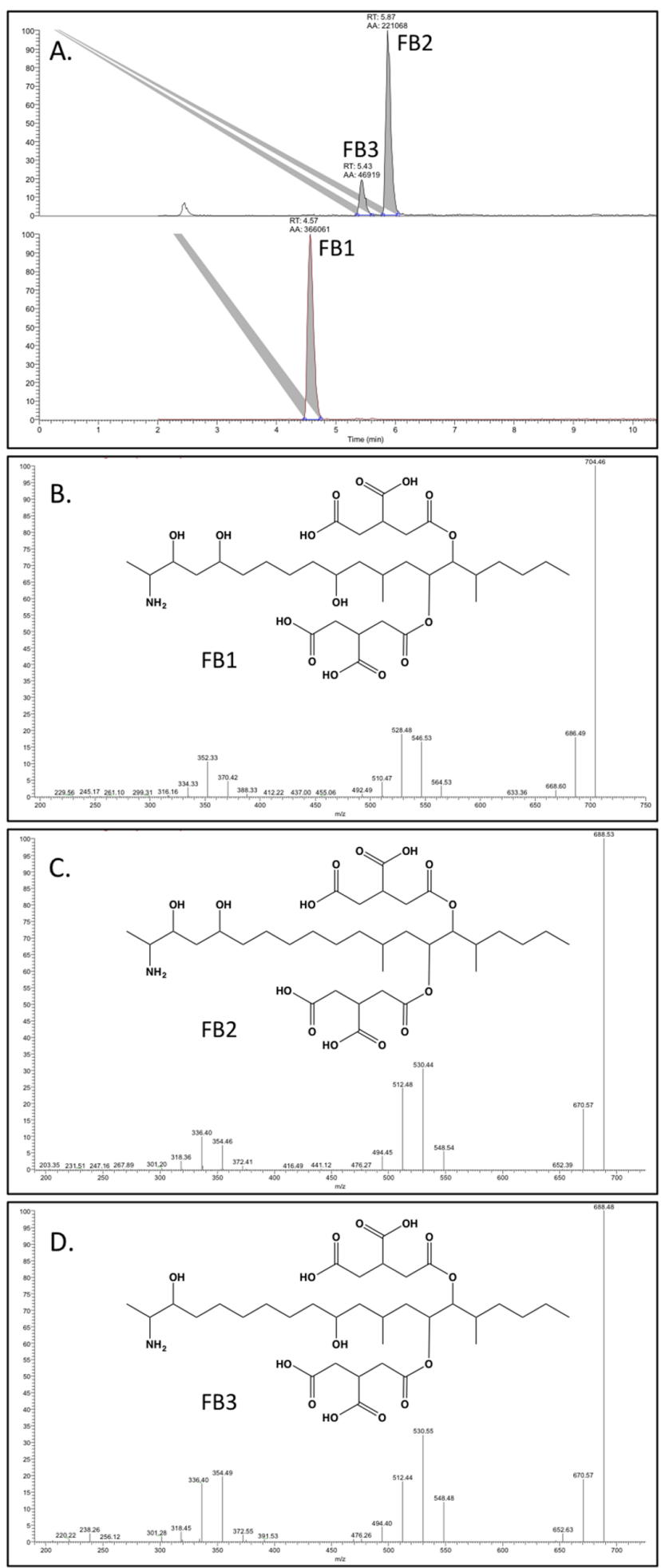

Figure 1. Liquid chromatography-tandem mass spectrometry (LC-MS/MS) analysis of fumonisins produced by F. fujikuroi winegrape isolate SLB 6214. (A) Chromatogram showing signature peaks for FB1, FB2, and FB3 with retention times of 4.57, 5.87, $5.43 \mathrm{~min}$, respectively. The structure and daughter ions $(\mathrm{m} / \mathrm{z}$ ) for (B) FB1; (C) FB2; and (D) FB3. 
Supplementary Materials: The following are available online at www.mdpi.com/2072-6651/8/9/254/s1.

Acknowledgments: The support for research throughout the southeastern wine industry is thoroughly appreciated, and the eight anonymous vineyard owners who graciously donated grape cluster samples during their busiest time of year attests to the sense of community in the southeastern U.S. wine region. The authors would like to thank William Gilbert, Cliff Knight, and Jack Yancey for laboratory support during fungal isolation and identification. Special thanks also to Trevor Mitchell, USDA-ARS-TMRU, for service with LC-MS/MS analysis, and Larry Pierce, USDA-ARS-TMRU, for technical support. Thanks to David M. Geiser of the Fusarium Research Center in Pennsylvania for TEF sequences of select reference strains. A grant from the Southeastern Small Fruit Consortium and scholarship funds from the American Wine Society Educational Foundation (AWS-North Alabama Chapter) greatly contributed to the financial support of this research.

Author Contributions: S.L.B., P.M.B., and A.E.G. conceived and designed the experiments; S.L.B. performed the experiments; S.L.B. and A.E.G. analyzed the data; P.M.B. contributed reagents/materials/analysis tools; S.L.B. wrote the paper.

Conflicts of Interest: The authors declare no conflict of interest.

\section{References}

1. Van der Merwe, K.J.; Steyn, P.S.; Fourie, L. Mycotoxins. Part II. The constitution of ochratoxins A, B, and C, metabolites of Aspergillus ochraceus Wilh. J. Chem. Soc. 1965. [CrossRef]

2. Gelderblom, W.C.; Jaskiewicz, K.; Marasas, W.F.; Thiel, P.G.; Horak, R.M.; Vleggaar, R.; Kriek, N.P. Fumonisins-novel mycotoxins with cancer-promoting activity produced by Fusarium moniliforme. Appl. Environ. Microbiol. 1988, 54, 1806-1811. [PubMed]

3. Zimmerli, B.; Dick, R. Ochratoxin A in table wine and grape-juice: Occurrence and risk assessment. Food Addit. Contam. 1996, 13, 655-668. [CrossRef] [PubMed]

4. Logrieco, A.; Ferracane, R.; Visconti, A.; Ritieni, A. Natural occurrence of fumonisin B2 in red wine from Italy. Food Addit. Contam. 2010, 27, 1136-1141. [CrossRef] [PubMed]

5. Mogensen, J.M.; Larsen, T.O.; Nielsen, K.F. Widespread occurrence of the mycotoxin fumonisin B2 in wine. J. Agric. Food Chem. 2010, 58, 4853-4857. [CrossRef] [PubMed]

6. Abarca, M.L.; Bragulat, M.R.; Castellá, G.; Cabanes, F.J. Ochratoxin A production by strains of Aspergillus niger var. niger. Appl. Environ. Microbiol. 1994, 60, 2650-2652. [PubMed]

7. Téren, J.; Varga, J.; Hamari, Z.; Rinyu, E.; Kevei, F. Immunochemical detection of ochratoxin A in black Aspergillus strains. Mycopathologia 1996, 134, 171-176. [CrossRef] [PubMed]

8. Heenan, C.N.; Shaw, K.J.; Pitt, J.I. Ochratoxin A production by Aspergillus carbonarius and A. niger and detection using coconut cream agar. J. Food Mycol. 1998, 1, 67-72.

9. WHO. Ochratoxin A. In Safety Evaluation of Certain Mycotoxins in Food, WHO Food Additives Series, 47, Proceedings of the 56th Meeting of the Joint FAO/WHO Expert Committee on Food Additives, Geneva, Switzerland, 6-15 February 2001; World Health Organisation: Geneva, Switzerland, 2001; pp. 281-387.

10. IARC. Monographs on the Evaluation of Carcinogenic Risk to Humans: Some Naturally Occurring Substances, Food Items and Constituents, Heterocyclic Aromatic Amines and Mycotoxins; International Agency for Research on Cancer, World Health Organization: Lyon, France, 1993; Volume 56, pp. 489-521.

11. Frisvad, J.C.; Smedsgaard, J.J.; Samson, R.A.; Larsen, T.O.; Thrane, U. Fumonisin B 2 production by Aspergillus niger. J. Agric. Food Chem. 2007, 55, 9727-9732. [CrossRef] [PubMed]

12. Logrieco, A.; Ferracane, R.; Haidukowsky, M.; Cozzi, G.; Visconti, A.; Ritieni, A. Fumonisin B2 production by Aspergillus niger from grapes and natural occurrence in must. Food Addit. Contam. 2009, 26, 1495-1500. [CrossRef] [PubMed]

13. Scott, P.M. Recent research on fumonisins: A review. Food Addit. Contam. 2012, 29, 242-248. [CrossRef] [PubMed]

14. IARC. Monographs on the Evaluation of Carcinogenic Risk to Humans: Some Traditional Herbal Medicines, Some Mycotoxins, Naphthalene and Styrene; International Agency for Research on Cancer, World Health Organization: Lyon, France, 2002; Volume 82, p. 301.

15. Oliveri, C.; Torta, L.; Catara, V. A polyphasic approach to the identification of ochratoxin A-producing black Aspergillus isolates from vineyards in Sicily. Int. J. Food Microbiol. 2008, 127, 147-154. [CrossRef] [PubMed]

16. Mikusova, P.; Srobarova, A.; Sulyok, M. Fusarium fungi and associated metabolites presence on grapes from Slovakia. Mycotoxin Res. 2013, 29, 97-102. [CrossRef] [PubMed] 
17. Mašková, Z.; Tančinová, D.; Rybárik, L.; Felšöciová, S. Colonization of grape berries by the genus Fusarium and toxigenity of the most common representatives. J. Microb. Biotech. Food Sci. 2014, 3, 256-258.

18. Tamura, M.; Takahashi, A.; Uyama, A.; Mochikizuki, N. A method for multiple analysis in wines by solid phase extraction and multifunctional cartridge purification, and ultra-high-performance liquid chromatography coupled to tandem mass spectrometry. Toxins 2012, 4, 476-486. [CrossRef] [PubMed]

19. Watanabe, M. Natural occurrence of fumonisin B1 in wine and fungal species causing the contamination in Japan. In Proceedings of the Annual Meeting of the United States Japan Cooperative Program on the Development and Utilization of Natural Resources, New Orleans, LA, USA, 25-30 January 2015.

20. Wiemann, P.; Sieber, C.M.K.; von Bargen, K.W.; Studt, L.; Niehaus, E.-M.; Espino, J.J.; Huß, K.; Michielse, C.B.; Albermann, S.; Wagner, D.; et al. Deciphering the cryptic genome: Genome-wide analyses of the rice pathogen Fusarium fujikuroi reveal complex regulation of secondary metabolism and novel metabolites. PLoS Pathog. 2013, 9, e1003475. [CrossRef] [PubMed]

21. Cruz, A.; Marín, P.; González-Jaén, M.T.; Aguilar, K.G.I.; Cumagun, C.J.R. Phylogenetic analysis, fumonisin production and pathogenicity of Fusarium fujikuroi strains isolated from rice in the Philippines. J. Sci. Food Agric. 2013, 93, 3032-3039. [CrossRef] [PubMed]

22. Desjardins, A.E.; Plattner, R.D.; Nelson, P.E. Production of fumonisin $B_{1}$ and monoliformin by Gibberella fujikuroi from rice from various geographic areas. Appl. Environ. Microbiol. 1997, 63, 1838-1842. [PubMed]

23. Glenn, A.E. Mycotoxigenic Fusarium species in animal feed. Anim. Feed Sci. Tech. 2007, 137, $213-240$. [CrossRef]

24. Rosler, S.M.; Sieber, C.M.K.; Humpf, H.-U.; Tudzynski, B. Interplay between pathway-specific and global regulation of the fumonisin gene cluster in the rice pathogen Fusarium fujikuroi. Appl. Microbiol. Biotechnol. 2016, 100, 5869-5882. [CrossRef] [PubMed]

25. Wulff, E.G.; Sørensen, J.L.; Lübeck, M.; Nielsen, K.F.; Thrane, U.; Torp, J. Fusarium spp. associated with rice Bakanae: Ecology, genetic diversity, pathogenicity and toxigenicity. Environ. Microbiol. 2010, 12, 649-657. [CrossRef] [PubMed]

26. Suga, H.; Kitajima, M.; Nagumo, R.; Tsukiboshi, T.; Uegaki, R.; Nakajima, T.; Kushiro, M.; Nakagawa, H.; Shimizu, M.; Kageyama, K.; et al. A single nucleotide polymorphism in the translation elongation factor $1 \alpha$ gene correlates with the ability to produce fumonisin in Japanese Fusarium fujikuroi. Fungal Biol. 2014, 118, 402-412. [CrossRef] [PubMed]

27. Desjardins, A.E.; Manandhar, H.K.; Plattner, R.D.; Manandhar, G.G.; Poling, S.M.; Maragos, C.M. Fusarium species from Nepalese rice and production of mycotoxins and gibberellic acid by selected species. Appl. Environ. Microbiol. 2000, 66, 1020-1025. [CrossRef] [PubMed]

28. Brannen, P.M.; Extension Fruit Disease Specialist. The University of Georgia, Athens, GA, USA. Personal communication, 2013.

29. Battilani, P.; Silva, A. Controlling ochratoxin A in the vineyard and winery. In Managing Wine Quality. Volume 1: Viticulture and Wine Quality; Reynold, A., Ed.; Woodhead Publishing in Food Science: Cambridge, UK, 2010; pp. 515-546.

30. Battilani, P.; Pietri, A.; Logrieco, A. Risk assessment and management in practice: Ochratoxin in grapes and wine. In Mycotoxins in Food: Detection and Control; Woodhead: Cambridge, UK, 2004; pp. 244-261.

31. Shim, W.B.; Woloshuk, C.P. Nitrogen repression of fumonisin B1 biosynthesis in Gibberella fujikuroi. FEMS Microbiol. Lett. 1999, 177, 109-116. [CrossRef] [PubMed]

32. King, A.D.; Hocking, A.D.; Pitt, J.I. Dichloran-rose bengal medium for the enumeration and isolation of molds from foods. Appl. Environ. Microbiol. 1979, 37, 959-964. [PubMed]

33. Glenn, A.E.; Hinton, D.M.; Yates, I.E.; Bacon, C.W. Detoxification of corn antimicrobial compounds as the basis for isolating Fusarium verticillioides and some other Fusarium species from corn. Appl. Environ. Microbiol. 2001, 67, 2973-2981. [CrossRef] [PubMed]

34. Ho, W.-C.; Ko, W.-H. A simple method for obtaining single-spore isolates of fungi. Bot. Bull. Acad. Sin. 1997, $38,41-44$.

35. Zhang, Y.J.; Zhang, S.; Liu, X.Z.; Wen, H.A.; Wang, M. A simple method of genomic DNA extraction suitable for analysis of bulk fungal strains. Lett. Appl. Microbiol. 2010, 51, 114-118. [CrossRef] [PubMed] 
36. O'Donnell, K.; Kistler, H.C.; Cigelnik, E.; Ploetz, R.C. Multiple evolutionary origins of the fungus causing Panama disease of banana: Concordant evidence from nuclear and mitochondrial gene genealogies. Proc. Natl. Acad. Sci. USA 1998, 95, 2044-2049. [CrossRef] [PubMed]

37. O'Donnell, K.; Ward, T.J.; Robert, V.A.R.G.; Crous, P.W.; Geiser, D.M.; Kang, S. DNA sequence-based identification of Fusarium: Current status and future directions. Phytoparasitica 2015, 43, 583-595. [CrossRef]

38. Fusarium MLST Database. Available online: http://www.cbs.knaw.nl/Fusarium (accessed on 1 July 2016).

39. Geiser, D.M.; Jimenez-Gasco, M.; Kang, S.; Makalowska, N.; Veeraraghavan, T.J.; Ward, N.; Zhang, G.A.; Kuldau, G.A.; O'Donnell, K. FUSARIUM-ID v.1.0: A DNA sequence database for identifying Fusarium. Eur. J. Plant Pathol. 2004, 110, 473-479. [CrossRef]

40. Altschul, S.F.; Gish, W.; Miller, W.; Myers, E.W.; Lipman, D.J. Basic local alignment search tool. J. Mol. Biol. 1990, 215, 403-410. [CrossRef]

41. Kearse, M.; Moir, R.; Wilson, A.; Stones-Havas, S.; Cheung, M.; Sturrock, S.; Buxton, S.; Cooper, A.; Markowitz, S.; Duran, C.; et al. Geneious Basic: An integrated and extendable desktop software platform for the organization and analysis of sequence data. Bioinformatics 2012, 28, 1647-1649. [CrossRef] [PubMed]

42. Agricultural Research Service Culture Collection. Available online: nrrl.ncaur.usda.gov (accessed on 1 July 2016).

Note: Author's contribution to the Work was done as part of the Author's official duties as a USDA employee and is a Work of the United States Government. Therefore, copyright may not be established in the United States.

(C) 2016 by the authors; licensee MDPI, Basel, Switzerland. This article is an open access article distributed under the terms and conditions of the Creative Commons Attribution (CC-BY) license (http://creativecommons.org/licenses/by/4.0/). 